\title{
ON AUTOMORPHISMS OF ALGEBRAIC GROUPS ${ }^{1}$
}

\author{
BY DAVID J. WINTER
}

Communicated by G. D. Mostow, February 10, 1966

Automorphisms of Lie algebras over fields of characteristic 0 have been investigated by Borel-Mostow, Jacobson, and Patterson (see [1], [4], [5]). This note describes some results in [6] of related investigations of automorphisms of algebraic groups over fields of arbitrary characteristic.

In the following discussions, $G$ is a connected linear algebraic group over an algebraically closed field of characteristic 0 or $p . \sigma$ and $\tau$ are (birational) automorphisms of $G$. The connected component of the identity of the group of fixed points of $\sigma$ is denoted by $F_{G}(\sigma)$.

$\sigma$ acts on the algebra $R(G)$ of representative functions of $G . \sigma$ is said to be algebraic if the orbit of each element of $R(G)$ under the cyclic group generated by $\sigma$ spans a finite dimensional subspace of $R(G)$.

An algebraic automorphism $\sigma$ is said to be semisimple (unipotent) if the induced transformation on $R(G)$ is semisimple (unipotent). If $\sigma$ is algebraic, $\sigma$ has a unique decomposition $\sigma=\sigma_{s} \sigma_{u}$ where $\sigma_{s}, \sigma_{u}$ are commuting algebraic automorphisms which are respectively semisimple and unipotent. If $G$ is semisimple, every (birational) automorphism of $G$ is algebraic (see [2], \$17-07).

If $\sigma$ is an algebraic automorphism of $G$, then there is a linear algebraic group $K$ containing $G$ as a closed normal subgroup and an element $s$ in $K$ such that $\sigma$ is the restriction to $G$ of the inner automorphism Ad $s$. If $\sigma$ is a semisimple (unipotent) algebraic automorphism of $G, s$ may be taken to be semisimple (unipotent); and such a $\sigma$ may be regarded as a semisimple (unipotent) element of $K$ by identifying $\sigma$ with such an $s$. On the other hand, elements $\sigma, \tau$ of $K$ are sometimes regarded as automorphisms of $G$ in the following discussions. (The above follows easily from results in [3].)

THEOREM 1. Let $\sigma$ and $\tau$ be semisimple elements of a linear algebraic group $K$ containing $G$ as a closed normal subgroup. Suppose that $\sigma G$ $=\tau G$. Let $H$ be a Cartan subgroup of $F_{G}(\sigma), L$ a Cartan subgroup of $F_{G}(\tau)$. Then there exists an element $g$ in $G$ such that $g^{-1} \mathrm{Hg}=L$ and $g^{-1} \sigma H g=\tau L$.

1 The work described here was done on a National Science Foundation Fellowship and is part of a dissertation directed by Professor George Seligman and presented for the degree of Doctor of Philosophy in Yale University. 
Sketch of Proof. By regarding $G$ as a transformation group on $\sigma G$ (an element $g$ in $G$ sending $\sigma x$ in $\sigma G$ in to $g^{-1} \sigma x g$ in $\sigma G$ ), it can be shown that the set $\left\{g^{-1} \sigma x g \mid g \in G, x \in 0_{H}\right\}$ is a dense épais subset of $\sigma G$, provided that $0_{H}$ is a dense épais subset of $H$. Such an $0_{H}$ can be chosen such that for $h$ in $0_{H}, H$ is the connected centralizer in $G$ of $\sigma h_{s}$ where $h_{s}$ is the semisimple part of $h$. (This and the preceding fact are established by applications of a transformation group formulation of Lemma $5, \S 6-11$ of [2].) There is a similar $0_{L}$ for $(\tau, L)$. Since two dense épais subsets of $\sigma G$ have nonempty intersection, there exist $g_{1}, g_{2}$ in $G, h$ in $0_{H}$ and $l$ in $0_{L}$ such that $g_{1}^{-1} \sigma h g_{1}=g_{2}^{-1} \tau l g_{2}$. Thus $g_{1}^{-1} \sigma h_{s} g_{1}=g_{2}^{-1} \tau l_{s} g_{2}$ and taking connected centralizers in $G$, $g_{1}^{-1} H g_{1}=g_{2}^{-1} L g_{2}$. The assertions of the theorem follow immediately.

Theorem 2. Suppose that $G$ is semisimple. Then if $\tau$ keeps stable a maximal torus $T$ and a Borel subgroup $B$ containing $T, F_{T}(\tau)$ contains a regular element of $G$ and is a Cartan subgroup of $F_{G}(\tau)$.

Proof. The cyclic group $A$ generated by $\tau$ acts in the group $T^{*}$ of rational characters of $T$ and keeps stable the subset $S$ of fundamental roots of $T$ with respect to $B$. Let $m$ be the index in $T^{*}$ of the subgroup generated by $S$. Assume that $\operatorname{dim} G>0$ and let $t$ be an element of $T$ of finite order such that $\alpha(t)=\beta(t)$ whenever $\alpha$ and $\beta$ are elements of $S$ which lie in the same orbit under $A$. Then $\alpha(\tau(t))=\alpha(t)$ for $\alpha$ in $S$. Thus $\chi^{m}(\tau(t))=\chi^{m}(t)$ for $\chi$ in $T^{*}$. Thus $\chi\left(\tau\left(t^{m}\right)\right)=\chi\left(t^{m}\right)$ for $\chi$ in $T^{*}$ and $\tau\left(t^{m}\right)=t^{m}$ since $T^{*}$ separates points. The order of $t$ (and hence of $\left.t^{m}\right)$ can be taken to be arbitrarily large. Thus $\operatorname{dim} F_{T}(\tau) \geqq 1$. Let $T_{1}=F_{T}(\tau)$ and let $G_{1}$ be the connected centralizer of $T_{1} . G_{1}$ is reductive with maximal torus $T$ and Borel subgroup $B \cap G_{1}$ (see [2]). $G_{1}$, $T$, and $B \cap G_{1}$ are $\tau$-stable. Thus if $\operatorname{dim} G_{1}^{(1)}>0$, an application of the above argument shows that $\operatorname{dim} F_{T_{2}}(\tau) \geqq 1$ where $T_{2}=T \cap G_{1}^{(1)}$. This is impossible since $F_{T_{2}}(\tau) \subseteq T_{1} \cap G_{1}^{(1)}$ and $T_{1} \cap G_{1}^{(1)}$ is finite. Thus $\operatorname{dim} G_{1}^{(1)}=0$. Thus $G_{1}=T$ and $F_{T}(\tau)$ contains a regular element of $G$. It now is immediate that $F_{T}(\tau)$ is a Cartan subgroup of $F_{G}(\tau)$.

THEOREM 3. Let $G$ be semisimple and let $\sigma$ be a semisimple (algebraic) automorphism of $G$. Let $T$ be a maximal torus of $G, B$ a Borel subgroup of $G$ containing $T$. Then there exists $g$ in $G$ such that $g^{-1} T g$ and $g^{-1} B g$ are stable under $\sigma . F_{G}(\sigma)$ contains a regular element of $G$.

Proof. Regarding $\sigma$ as a semisimple element of an algebraic linear group $K$ containing $G$ as a closed normal subgroup, choose a semisimple element $\tau$ of $\sigma G$ such that Ad $\tau$ keeps stable $T$ and $B$ (possible by the conjugacy of maximal tori and Borel subgroups under inner automorphisms). Then $\sigma G=\tau G$ and $F_{T}(\tau)$ is a Cartan subgroup of 
$F_{G}(\tau)$ (Theorem 2). Thus letting $H$ be a Cartan subgroup of $F_{G}(\sigma)$, there exists $g$ in $G$ such that $g^{-1} F_{T}(\tau) g=H$ and $g^{-1} \tau F_{T}(\tau) g$ contains $\sigma$ (Theorem 1). For such a $g, g^{-1} T g, g^{-1} B g$ are $\sigma$-stable since $T, B$ are $\tau$-stable. An application of Theorem 2 now shows that $F_{G}(\sigma)$ contains a regular element of $G$.

Theorem 3, along with Theorem 2 and the methods used in its proof, can be used to compute the rank of $F_{G}(\sigma)$ where $\sigma$ is a semisimple algebraic automorphism of a semisimple algebraic group $G$ (the rank of $F_{G}(\sigma)$ corresponds to the index of " $\sigma G$ " in [4]).

A straightforward consequence of the preceding theorem is

CoRollary 4. Let $\sigma$ be a semisimple algebraic automorphism of $G$. Then

(1) $\sigma$ keeps stable a Borel subgroup of $G$;

(2) $\sigma$ keeps stable a maximal torus of $G$;

(3) the centralizer in $G$ of a maximal torus in $F_{G}(\sigma)$ is solvable.

R. Steinberg has independently proved part (1) of Corollary 4, using methods which require only that one assume that $\sigma$ be a birational automorphism of $G$.

The proofs of the following two theorems will appear in a later paper.

TheOREM 5. If $\sigma$ has only finitely many fixed points, then $G$ is solvable.

ThEOREM 6. Suppose that $\sigma$ has finite order $n$ and that $\sigma$ has only finitely many fixed points in $G$. Then $\sigma$ keeps stable precisely one maximal torus $T_{\sigma}$ of $G$, and the fixed points of $\sigma$ are elements of $T_{\sigma}$ whose orders divide $n$. If $n$ is prime, $G$ is nilpotent.

\section{REFERENCES}

1. A. Borel and G. D. Mostow, On semi-simple automorphisms of Lie algebras, Ann. Math. 61 (1955), 389-504.

2. C. Chevalley, Classification des groupes de Lie algébriques, Séminaire C. Chevalley, Vols. I, II, Ecole Norm. Sup. Paris, 1958.

3. G. Hochschild and G. D. Mostow, Representations and representative functions of Lie groups, Ann. Math. 66 (1957), 495-452.

4. N. Jacobson, $A$ note on automorphisms of Lie algebras, Pacific J. Math. 12 (1962), 303-315.

5. E. M. Patterson, On regular automorphisms of certain classes of rings, Quart. J. Math. 12 (1961), 127-133.

6. D. J. Winter, On automorphisms of Lie algebras and algebraic groups, Doctoral Dissertation, Yale University, New Haven, Conn., 1965.

YaLE UNIVERSITY 